\title{
RECOGNITION AND RECONSTRUCTION OF LATE AUDITORY EVOKED POTENTIALS USING WAVELET ANALYSIS
}

\author{
S. Weiß \\ Signal Processing Division \\ Dept. of Electronic and Electrical Eng. \\ University of Strathclyde \\ Glasgow, Scotland \\ Email: weiss@spd.eee.strath.ac.uk
}

\author{
U. Hoppe \\ Abteilung für Phoniatrie und Pädaudiologie \\ Universitäts-HNO-Klinik \\ Friedrich-Alexander Universität \\ Erlangen, Germany \\ Email: uhoppe@lte.e-technik.uni-erlangen.de
}

\section{ABSTRACT}

In this paper we introduce a recognition and reconstruction method for late auditory evoked potentials (AEPs) using wavelet analysis. AEPs are part of the Electroencephalogram (EEG) in reaction to acoustic stimuli and are used for diagnostic purposes. The decomposition of the EEG measurement data into subbands can yield a parameterization of the AEP using a small number of significant coefficients, which can be determined by surveying distributions of the transform coefficients. This method is more reliable than traditional means, and additionally offers the possibility of reconstructing potential AEPs by performing an inverse transform on significant coefficients.

\section{INTRODUCTION}

In audiometry, auditory evoked potentials (AEPs) are used to obtain an objective audiogram. $\mathrm{AEP}_{\text {s }}$ form part of the Electroencephalogram (EEG) as reaction to an acoustic stimulus, and can therefore represent hearing ability independently of the active cooperation or subjective impression of the experimentee [1]. Within the AEP, only the frequency specific late reactions from the cortex are of interest, appearing $20 \mathrm{~ms}$ to $400 \mathrm{~ms}$ after the stimulus has been given. The main inconvenience of late AEPs is the background noise in the EEG caused by other nervous processes in the cortex, resulting in an extremely poor SNR. Further problems are raised by the non-stationarity of the AEP, its dependence on the tone level and differences between individuals. Referring to an objective audiogram, this objectivity is only related to the experimentee, as the data still has to be evaluated by an operator and thus a decision is based on his experience of recognizing certain features within the curves, and the quality of the data.

The main effort in research of late AEPs is therefore to find parameters and methods that help indicate the presence of an AEP in the EEG. Traditional methods range from synchronous averaging and calculating of cross-correlations [1] to methods using Fourier coefficients, cross-correlation coefficients of partial averages, power ratio of the normal average compared to an average, where every second measurement is weighted with a minus sign (response to noise ratio), and the cross-correlation of the ensemble average with a given pattern, as described in. Hoth et al. [2],[3]

Bartnik et al. [4] were the first to apply a discrete wavelet transform (DWT) to reconstruct evoked potentials. Having calculated the transform coefficients of a number of EEG measurements, they identify five coefficients with which a reconstruction of the suspected contained evoked potential is then performed, and an intuitive comparison with the ensemble average is done. The main drawbacks of their work are the assumption that the basic parameters of the potential can be identified using always the same five coefficients, a very small set of measurements to check the reliability of their method, and no quantitative assessment of the reconstructed data.

Based on the idea of Bartnik et al. of calculating the DWT of EEG measurements and on some knowledge of AEPs and EEG measurements described in Sec. 2, we derive a method for identifying transform coefficients that are "significant" for an AEP, where this significance is evaluated statistically [5]. The decision of whether an AEP is contained in the EEG or not is then based on the presence of any significant coefficients. Afterwards, a reconstruction can be performed by applying an inverse DWT to the coefficients that have been assigned significance. This is subject of Sec. 3, while Sec. 4 draws conclusions and discusses the practical value of the presented method.

\section{CHARACTERISTICS OF LATE AUDITORY EVOKED POTENTIALS}

A late AEP appears as a low frequency wave of $3-8 \mathrm{~Hz}$ [2] in the EEG. Its maximum is usually around $100 \mathrm{~ms}$ after the stimulus, although this latency varies for different tone levels, and can even change slightly within a set of measurements. As the tone level approaches the hearing threshold, the latency becomes larger and the amplitude diminishes, as it can be seen in the ensemble-averaged EEG measurements Fig. 1, which correspond to a person with normal hearing.

The shape of the AEP varies greatly between individuals, particularly if their brains are not fully developed, eg for children who show very large latencies. In the clinical practice, the majority of the cases exhibits an unusual AEP pattern that is difficult of detect for an operator, and thus a recognition method is most wanted. Therefore, any method should be based on as few assumptions as possible. 

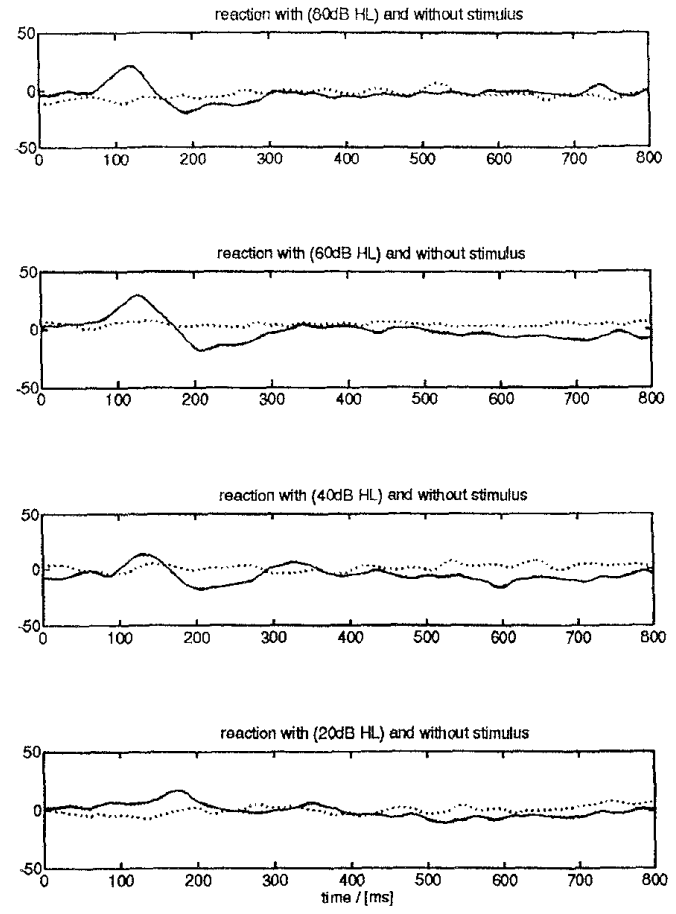

Figure 1: averages of 100 sweeps each, with (solid) and without (dotted) given stimulus (sine-burst of $1 \mathrm{kHz}$ ).

The EEG for our work is a measured voltage between forehead and mastoid, which is pre-processed and sampled at $640 \mathrm{~Hz}$. Synchronized to a stimulus, a sequence of $800 \mathrm{~ms}$ is recorded, yielding 512 samples. In the following sections, such a single synchronous sequence is termed sweep.

\section{WAVELET ANALYSIS OF EEG MEASUREMENT'S}

\subsection{Applying the Wavelet Transform}

A dyadic wavelet decomposition of sweeps uses Mallat's pyramidal algorithm for multi-resolution analysis [6]. It is based on two sets of subspaces of $\mathbb{L}^{2}(\mathbf{R}), \mathbf{V}_{2^{k}}$ and $\mathbf{O}_{2^{k}}$, where $\ldots \mathbf{V}_{2^{k+1}} \supset \mathbf{V}_{2^{k}} \supset \mathbf{V}_{2^{k-1}} \ldots$ and $\mathbf{O}_{2^{i}} \cap \mathbf{O}_{2^{k}}=\emptyset$, $\forall i \neq k \in \mathbf{Z} . \mathbf{V}_{2^{k}}$ and $\mathbf{O}_{2^{k}}$ are mutually exclusive, $\mathbf{V}_{2^{k}} \cap$ $\mathbf{O}_{2^{k}}=\emptyset$, and their sum is dense in $\mathbf{V}_{2^{k+1}}, \mathbf{V}_{2^{k}} \cup \mathbf{O}_{2^{k}}=$ $\mathbf{V}_{2^{k+1}}, \forall k \in \mathbf{Z}$. Furthermore, $\left.\mathbf{V}_{2^{k}}\right|_{k \rightarrow \infty}$ is dense in $\mathbb{L}^{2}(\mathbf{R})$. Orthonormal bases for the spaces $\mathrm{V}_{2^{k}}$ and $\mathbf{O}_{2^{k}}$ are spanned by wavelets $\psi_{k, n}(t)=2^{k / 2} \psi\left(2^{k}(t-n)\right)$ and scaling functions $\phi_{k, n}(t)=2^{k / 2} \phi\left(2^{k}(t-n)\right)$, respectively, for $k, n \in \mathbf{Z}$.

A signal $f(t)$ can be projected onto these subspaces of $\mathbb{L}^{2}(\mathbf{R})$ by weighting the basis functions with coefficients $\alpha_{k}(n)$ and - the wavelet coefficients - $\beta_{k}(n)$, which are calculated by scalar products of $f(t)$ with $\psi_{k, n}(t)$ and $\phi_{k, n}(t)$, respectively, yielding functions $A_{2^{k}} f(t)$ and $D_{2^{k}} f(t)$,

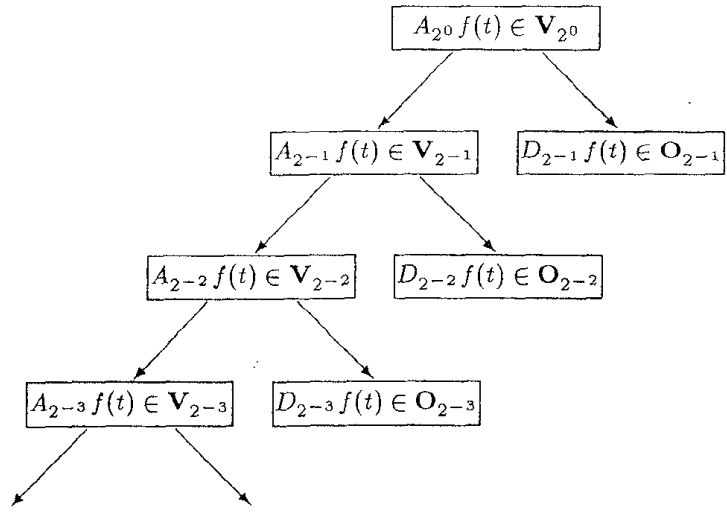

Figure 2: pyramidal decomposition of a signal into coarser approximations $A_{2^{k}}$ and details $D_{2^{k}}$ (Mallat). The starting sequence is $A_{2^{\circ}} f(t)$.

where $A_{2^{k}} f(t) \in \mathrm{V}_{2^{k}}$ is a coarse approximation of $f(t)$ and $D_{2^{k}} f(t) \in \mathrm{O}_{2^{k}}$ is the detail information that is lost when going from a less coarse approximation $A_{2^{k+1}} f(t)$ to $A_{2^{k}} f(t)$.

Mallat [6] derives an iterative computation of coefficients: starting from a coefficient set $\alpha_{0}(n)$ of $A_{2^{\circ}} f(t)$, subsequently $\alpha_{k}(n)$ of coarser approximations can be obtained by lowpass filtering of the coefficients, while the wavelet coefficients $\beta_{k-1}(n)$ are yielded by a highpass filter operation on $\alpha_{k}(n)$. The filter coefficients can be calculated in dependence on $\psi(t)$ and $\phi(t)$. The resulting pyramidal scherne for the computation of the wavelet coefficients is shown in Fig. 2. It can be implemented as a critically decimated octave filter bank. As the bases of $\mathbf{V}_{2^{k}}$ and $\mathbf{O}_{2^{k}}$ are orthonormal, a unique inverse transform exists, which is calculated by going through the pyramidal algorithm in a reverse direction.

A start sequence $\alpha_{0}(n)$ of $A_{2^{\circ}} f(t)$ should ideally be computed by scalar products of the continuous time signal, $f(t)$, with translated scaling functions $\phi_{0, n}(t-n), n \in \mathbf{Z}$. As the time duration of $\phi(t)$ is comparably short to the wave lengths of interest, and because of $\int_{-\infty}^{\infty} \phi(t) d t=1$ [7], we can intuitively view $\phi(t)$ as a Dirac impulse, such that the start sequence is simply the sampled discrete time version of $f(t)$.

Some problems arise, as the sweeps are finite intervals and important low frequency information is contained close to the beginning of a sweep. The analysis of the low frequencies requires a filter bank of considerable depth, such that with zero-padding most of the important features are distorted by transients. If the sweeps are extended periodically, the filters are opexated in steady state, distortions are suppressed and the periodicity is preserved in the output data. Circular convolution has the disadvantage of discontinuity, which causes leakage of information from the end to the beginning of the sweeps, and vice versa. Best results could be obtained using a symmetric extension, where the resulting waveform is symmetric to the end points of the 

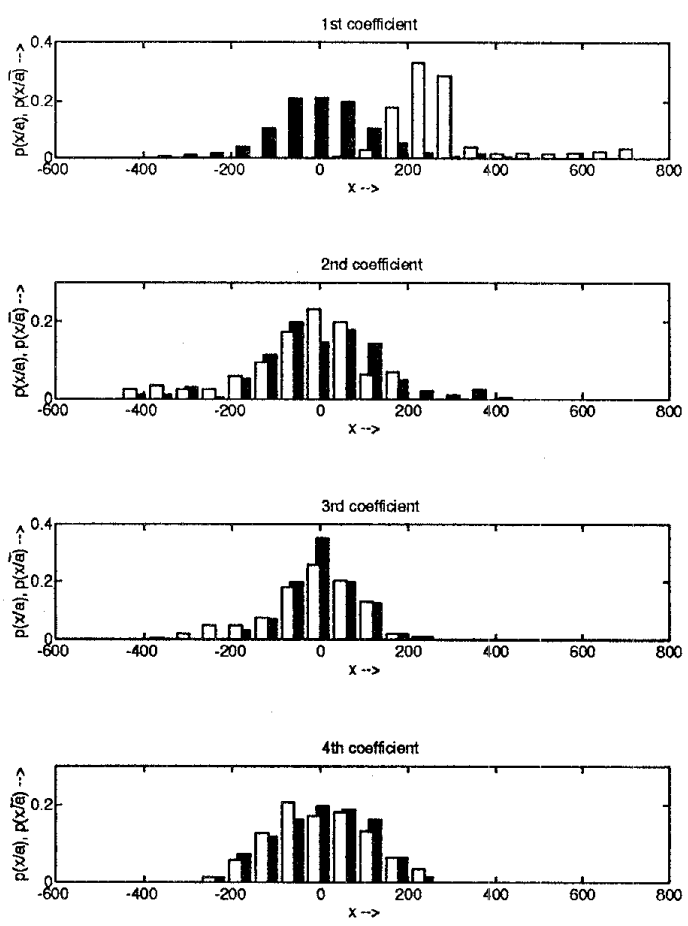

Figure 3: relative frequency distribution of wavelet coefficients of detail $D_{2-7}$. The white bars refer to a given stimulus, while filled bars mark sweeps without AEP.

sweep sequence, although this imposes the restriction to use symmetric wavelets and hence linear phase filters only [8], and has slight implications for the linearity of the filter bank operation and the validity of Parseval's Theorem [5]. Apart from the symmetric wavelet described by Mallat [6], symmetric biorthogonal wavelets listed in [8] and [9] have been used for the analysis, but however give poorer results.

\subsection{Processing of Transform Coeflicients}

The reconstruction of single evoked potentials as proposed in [4] from certain transform coefficients proved useless for late AEPs because of their poor SNR. Father than basing a method on a single sweep, we fall back upon the statistics of a number of successive sweeps.

Fig. 3 shows the relative frequency distribution of the four wavelet coefficients in detail $D_{2-7}$ measured from 100 sweeps, where blanc and filled bars refer to sweeps with and without given stimulus, respectively. Obviously, the statistics of the first coefficient indicates a difference between sweeps with a suspected AEP and sweeps that cannot contain an AEP, while the distributions of the later three coefficients are rather indifferent. It can therefore be concluded, that the first wavelet coefficient in contrary to the others in $D_{2-7}$ must be "significant" for an AEP.
To yield a qualitative measure for this intuitive difference of distributions, we define a selectivity value. Based on two different conditional probability densities $p(x \mid a)$ and $p(x \mid \bar{a})$, where the random variable $x$ is the value of the wavelet coefficient given that either a stimulus of a certain level $(a)$ or no stimulus $(\bar{a})$ has been presented to the experimentee prior to recording a sweep, this value is chosen according to Fig. 4. Assuming for the mean values $\mu_{x \mid a} \geq \mu_{x \mid \bar{a}}$ w.l.o.g., we measure the distributions $P(x \mid a)$ and $1-P(x \mid \bar{a})$ for every coefficient. By determining the intersection of both distributions, we find the optimum decision threshold $x_{\text {opt }}$, at which we yield the total error to be $e=(\alpha+\beta) / 2$, where $\alpha$ and $\beta$ are the likelihoods for a false decision for $a$ or $\bar{a}$, under the assumption of equal likely $a$ and $\bar{a}$. In case $\mu_{x \mid a}<\mu_{x \mid \bar{a}}$, the total error can be calculated from the intersection value $e^{\prime}$ such that $e=1-e^{\prime}$.

The selectivity value now is this total minimum value, which covers the interval $[0,0.5]$, with small values referring to a good separability of the distribution, while values close to 0.5 indicate indifferent distributions. By setting a threshold for this selectivity value, wavelet coefficients can be distinguished by their relative frequencies to be "significant" or "insignificant" in contributing to an AEP. If the threshold is passed for any of the coefficients, an AEP is assumed in the EEG.
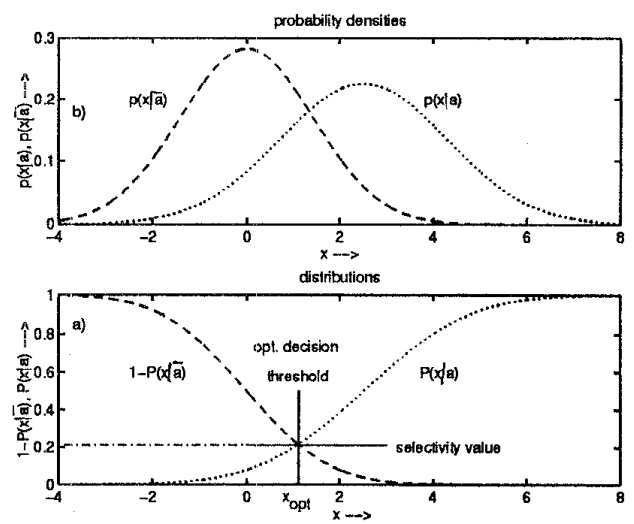

Figure 4: two different probability densities (a) and the according distributions (b), from which the selectivity value is derived graphically.

\subsection{Test Results and Reconstruction}

Tests on EEG measurement series obtained from 40 experimentees have been carried out, of which about half the measurements have recorded the EEG without any given stimulus. Significant transform coefficients could almost only be found in the details $D_{2-6}$ (4 coef., frequency band approx. $5-10 \mathrm{~Hz})$ and $D_{2-7}$ (8 coef., frequency band approx. $2.5-5 \mathrm{~Hz}$ ), such that the examination of distributions can be restricted to these 12 coefficients, which agrees with the frequency range reported in [2]. A comparison with methods described in Hoth et al. $[2,3]$ is given in the following table 

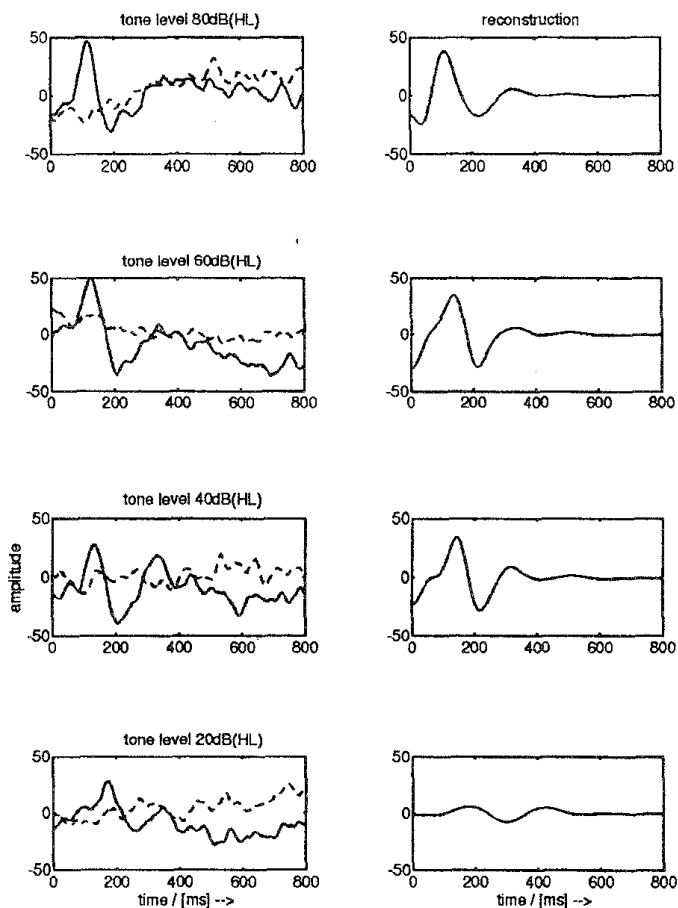

Figure 5: ensemble averages compared to reconstruction with relevant coefficients

along with the minimum false decision rate, where the decision threshold has been adjusted optimally by an approach analogue to Fig. 4.

\begin{tabular}{|l|c|}
\hline method & false decision rate \\
\hline \hline Fourier coef. at $5 \mathrm{~Hz}$ & 0.15 \\
\hline response to noise ratio & 0.27 \\
\hline correlation of partial averages & 0.20 \\
\hline selectivity of wavelet coef. & 0.09 \\
\hline
\end{tabular}

For the measurements at hand the proposed method obviously outperforms the others in terms of the most reliable decision. Furthermore, a reconstruction based on coefficients marked as significant can be done by performing an inverse DWT on the mean value of their measured distributions, as shown in Fig. 5 .

\section{CONCLUSION}

A new method for the recognition of late AEPs has been presented. The DWT offers a good parameterization of AEPs by a small number of coefficients, which is exploited to identify these coefficients by their distribution.

The examination of distributions includes 12 coefficients and is free of any assumptions conceming their distributions or the latency of the AEP. The restriction to a lim- ited frequency band is reasonable, as it has little effect on the recognition and improves speed greatly, such that data. can be evaluated on-line to the measurements. A new possibility compared to other AEP indication methods is the reconstruction with significant coefficients by means of an inverse transform.

Although an automatic recognition is still not possible, as parameters like the threshold for decisions have to be chosen by an operator, as it has to be done with all traditional means, the operator is given an additional support for his or her decision.

Current research examines the use of sequential testing [10] to be included, which will add an analytic measure for the statistical reliability and is thus expected to shorten the time of examinations, which would be very beneficial to patients.

\section{ACKNOWLEDGEMENTS}

The authors would like to thank T. Sporer, Lehrstuhl für Technische Elektronik, and M. Moser, Abteilung für Phoniatrie und Pädaudiologie, both at Friedrich-Alexander Universität Erlangen-Nürnberg, for helpful comments, discussions, and technical support. This work is supported by a grant from Friedrich-Alexander Universität, Erlangen.

\section{REFERENCES}

[1] G. H. Steeger. Ein Beitrag zur Verbesserung der Messung akustisch evozierter Potentiale variabler Latenz im Elektroenzephalogramm des Menschen. Dissertation, Universität Erlangen-Nürnberg, Erlangen, 1979.

[2] S. Hoth and C. Weber. "Kritische Wertung der Hörschwellenbestimmung mittels der Hirnrindenpotentiale, Teil 1". Audiol. Akustik, pp.190-200, May 1990.

[3] S. Hoth and C. Weber. "Kritische Wertung der Hörschwellenbestimmung mittels der Hirnrindenpotentiale, Teil 2". Audiol. Akustik, pp.244-256, June 1990.

[4] E. Bartnik, K. Blinowska, and P. Durka. "Single evoked potential reconstruction by means of wavelet transform". Biol. Cybern., 67:pp.175-181, 1992.

[5] S. Weiß. Rekonstruktion Akustisch Evozierter Potentiale. Diplomarbeit, Universität Erlangen-Nürnberg, Erlangen, Dec. 1994.

[6] S. G. Mallat. "A Theory for Multiresolution Signal Decomposition: The Wavelet Representation". IEEE Trans. Pattern Anal. Machine Intell, 11(7):pp.674692, July 1989.

[7] P. Vaidyanathan. Multirate Systems and Filter Banks. Prentice Hall, 1993.

[8] I. Daubechies. Ten Lectures on Wavelets. SIAM, Philadelphia, 1992.

[9] M. Vetterli and C. Herley. "Wavelets and Filter Banks: Theory and Design". IEEE Trans. SP, 40(9):pp.22072232, Sept.1992.

[10] R. G. Miller. "Sequential Signed-Rank Test". J. Amer. Stat. Assoc., 65(332):pp.1554-1561, Dec. 1970. 